Agriculture

Agriculture fields

Okayama University

Year 1999

\title{
Changes in intracellular content of glutathione and thiols aociated with $\gamma$-glutamyl cycle during sperm penetration and pronuclear formation in rat oocytes
}

\author{
Hiroaki Funabashi Naoya Bandoh Shinobu Nakahira \\ Okayama University Okayama University Okayama University \\ She-hoon $\mathrm{Oh}$ \\ Seiji Tsuboi \\ Okayama University Okayama University
}

This paper is posted at eScholarship@OUDIR : Okayama University Digital Information Repository.

http://escholarship.lib.okayama-u.ac.jp/agriculture_general/2 


\title{
Changes in intracellular content of glutathione and thiols associated with $\gamma$-glutamyl cycle during sperm penetration and pronuclear formation in rat oocytes
}

\author{
Hiroaki Funahashi ${ }^{1}$, Naoya Bandoh ${ }^{2}$, Shinobu Nakahira ${ }^{1}$, She-Hoon Oh ${ }^{1}$ and Seiji Tsuboi ${ }^{2}$ \\ Department of Animal Science and Technology, and Department of Physiological Chemistry, Okayama University, \\ Okayama, Japan
}

Date submitted: 20.4.99. Date accepted: 17.6.99

\section{Summary}

The content of glutathione and other thiols in rat eggs was examined during sperm penetration and pronuclear formation by high-performance liquid chromatography with fluorescence detection. Reduced glutathione (GSH) content was higher in unfertilised oocytes $(8.50 \pm 0.29 \mathrm{pmol} / \mathrm{egg})$ and penetrated eggs with a decondensed sperm nucleus (DSH eggs; $7.72 \pm 0.56 \mathrm{pmol} / \mathrm{egg}$ ) than eggs at the pronuclear stage (PN eggs; $5.93 \pm 0.10 \mathrm{pmol} / \mathrm{egg}$ ). The content of oxidised glutathione (GSSG) was not different among experimental groups (152.6 $\pm 74.1 \mathrm{nmol} / \mathrm{egg}$ in unfertilised eggs, $146.0 \pm 50.0 \mathrm{nmol} / \mathrm{egg}$ in DSH eggs and $39.7 \pm 17.3 \mathrm{nmol} / \mathrm{egg}$ in PN eggs). The GSSG/GSH ratio did not change during fertilisation. Although the reduced cysteinylglycine content of eggs did not change among experimental groups, the oxidised form of cysteinylglycine increased $(p<0.025)$ between sperm decondensation $(6.9 \pm 1.5$ $\mathrm{nmol} / \mathrm{egg}$ in unfertilised oocytes and $10.1 \pm 2.1 \mathrm{nmol} / \mathrm{egg}$ in DSH eggs) and pronuclear formation (40.5 $\pm 11.5 \mathrm{nmol} / \mathrm{egg}$ in PN eggs). Low contents of cystine were detected during fertilisation but cysteine and $\gamma$-glutamylcysteine were not detected in any treatment groups. These results demonstrate that GSH content in rat eggs decreases between sperm decondensation and pronuclear formation, probably due to the increased activity of $\gamma$-glutamyl transpeptidase.

Key words: Decondensation of spermatozoa, Fertilisation, Glutathione, Pronuclear formation, Rat eggs

\section{Introduction}

Glutathione, the major intracellular free thiol, and other thiols in the cytoplasm are the most likely candidates for the oocyte to reduce disulphide bonds in the nuclear protamine of penetrating spermatozoa (Perreault, 1990; Yanagimachi, 1994). A high content of a reduced form of glutathione (GSH) at sperm penetration appears to be beneficial for successful sperm decondensation and male pronuclear formation (Per-

All correspondence to: H. Funahashi, Department of Animal Science and Technology, Faculty of Agriculture, Okayama University, Tsushima-naka, Okayama 700-8530, Japan.

${ }^{1}$ Department of Animal Science and Technology, Faculty of Agriculture, Okayama University, Tsushima-naka, Okayama 700-8530, Japan.

${ }^{2}$ Department of Physiological Chemistry, Faculty of Pharmaceutical Sciences, Okayama University, Tsushima-naka, Okayama 700-8530, Japan. reault et al., 1988; Yoshida, 1993). Intracellular levels of GSH and glutathione disulphide (GSSG; oxidised form of GSH) increase during maturation following germinal vesicle breakdown and are lower in fertilised eggs at the pronuclear stage when compared with mature oocytes in the hamster and pig (Perreault et al., 1988; Yoshiga et al., 1993; Funahashi et al., 1995). In porcine eggs, total GSH and GSSG content decreased following sperm penetration and prior to sperm decondensation (Funahashi et al., 1995). Recently, it has been suggested that $\gamma$-glutamyl transpeptidase (GGT) present on the surface of boar spermatozoa is associated with a decrease in oocyte glutathione (GSH + GSSG) content at fertilisation (Funahashi et al., 1996). GGT is a membrane-binding enzyme associated with degradation of glutathione in the $\gamma$-glytamyl cycle (Meister et al., 1976; Tate \& Meister, 1981).

However, the time at which oocyte glutathione content decreases following sperm penetration is not 
known in other mammalian species, nor is the levels of other thiols in eggs or the changes in these during fertilisation. Recently a methodology has been developed for the simultaneous detection of thiols and disulphides by high-performance liquid chromatography (HPLC) with fluorescence detection (Toyo'oka et al., 1988). The present study was designed to determine the change in intracellular content of glutathione and other thiols in rat eggs during sperm penetration and pronuclear formation by HPLC with modified fluorescence detection.

\section{Materials and methods}

\section{Collection of ovulated oocytes and penetrated eggs}

Sexually mature female Wistar rats (2-3 months old) were maintained under controlled lighting conditions (14L:10D; lights on at 0600 hours). For collection of ovulated oocytes, rats were killed by cervical dislocation at 1000 hours on the day of oestrus, which was identified by examination of vaginal smears. To obtain penetrated eggs, rats at pro-oestrus, which was identified by examination of vaginal smears, were naturally mated overnight with males of the same strain. On the following morning females were examined for the presence of vaginal plugs or spermatozoa in the vagina. To collect eggs with decondensed sperm head (DSH eggs) and zygotes at the pronuclear stage (PN eggs), mated rats were killed by cervical dislocation at 0800 and 1500 hours, respectively. Eggs were recovered by flushing the excised oviducts with approximately $0.1 \mathrm{ml}$ of HEPES-buffered Tyrode's lactate medium containing $0.1 \%$ polyvinylalcohol (TLHEPES-PVA) supplemented with $0.1 \%$ hyaluronidase through the fimbrial opening. Eggs were denuded by pipetting and then washed three times with the medium without hyaluronidase. Under a dissecting microscope, only those eggs in which a sperm head was not visible but a penetrating sperm tail existed, or in which pronuclei were visible, were selected as DSH eggs and PN eggs, respectively. Five microlitres of TL-HEPES-PVA containing 40-50 eggs was transferred to a $1.5 \mathrm{ml}$ microcentrifuge tube and $5 \mu \mathrm{l}$ of $1.25 \mathrm{M}$ phosphoric acid added to the tube. After making sure of the disappearance of the zona pellucida and plasma membrane under a stereomicroscope, the tubes containing samples were stored in a freezer at $-80{ }^{\circ} \mathrm{C}$ until use for HPLC with fluorescence detection.

\section{Evaluation of sperm penetration in DSH eggs}

To make sure the DSH eggs contained a decondensed sperm head, some of these were examined under a phase-contrast microscope after fixation in $10 \%$ neutral formalin for 4-6 h, dehydration with $95 \%$ ethanol, and staining with $0.25 \%$ lacmoid in $45 \%$ acetic acid. To assess what percentage of sperm tails had been incorporated in the DSH eggs, some of the eggs were freed from the zona pellucida and decorating spermatozoa by treatment in $0.1 \%$ proteinase in Dulbecco's phosphate-buffered saline (PBS). The zona free DSH eggs were washed three times with fresh Dulbecco's PBS supplemented with $0.4 \%$ bovine serum albumin (BSA), and then examined using a dissecting microscope.

\section{HPLC separation of thiols and disulphides in unpenetrated and penetrated eggs}

The contents of thiols and disulphides associated with the $\gamma$-glutamyl cycle were determined by HPLC after labelling with 4-(aminosulphonyl)-7-fluoro-2,1,3benzoxadiazole (ABD-F, Dojindo Laboratories, Kumamoto, Japan) as the precolumn derivative reagent for thiols and ammonium 7-fluoro-2,1,3-benzoxadiazole-4-sulphonate (SBD-F, Dojindo Laboratories, Kumamoto, Japan) for disulphides (Toyo'oka et al., 1988) with modification. Briefly a $50 \mu$ l portion of $0.5 \%$ ABD-F in $0.7 \mathrm{M}$ sodium borate buffer ( $\mathrm{pH}$ 9.3) containing $5 \mathrm{mM}$ EDTA was added to the sample tubes, heated at $60{ }^{\circ} \mathrm{C}$ for $5 \mathrm{~min}$, and then extracted three times with $1 \mathrm{ml}$ of ethyl acetate on ice. The aqueous layer was treated at $60^{\circ} \mathrm{C}$ for 20 min with $5 \mu$ of $5 \%$ SBD-F in 0.7 M sodium borate buffer ( $\mathrm{pH}$ 9.3) containing $5 \mathrm{mM}$ EDTA and $5 \mu \mathrm{l}$ of $10 \%$ tri- $n$-butylphosphine in isopropanol/acetonitrile $(2 / 98, \mathrm{v} / \mathrm{v})$. After cooling on ice, $10 \mu \mathrm{l}$ of $4 \mathrm{~N} \mathrm{HCl}$ was added, and then the final solution was subjected to HPLC.

The analytical column used for HPLC was ODS80Ts (Toso, Tokyo, Japan). A fluorescence detector (RF10A-XL, Shimadzu, Kyoto, Japan) was used for detection with an excitation wavelength of $380 \mathrm{~nm}$ and an emission wavelength of $510 \mathrm{~nm}$. The data were calculated by an integrator (C-R6A chromatopac, Shimadzu, Kyoto, Japan). The flow rate of the eluent was fixed at $1.0 \mathrm{ml} / \mathrm{min}$ by a controller (LC-10AT, Shimadzu, Kyoto, Japan). The separation of the derivatives was achieved in a linear gradient elution from $1 \%$ acetonitrile in $75 \mathrm{mM}$ sodium citrate ( $\mathrm{pH} 2.9$ ) to $4 \%$ for $2 \mathrm{~min}$, an isocratic elution of $4 \%$ acetonitrile in $75 \mathrm{mM}$ sodium citrate ( $\mathrm{pH}$ 2.9) for $6 \mathrm{~min}, 7 \%$ acetonitrile (in $75 \mathrm{mM}$ sodium citrate, $\mathrm{pH} 2.9$ ) for $12 \mathrm{~min}$ and then a linear gradient elution from $7 \%$ acetonitrile to $15 \%$ for $30 \mathrm{~min}$. For calibration, a solution spiked with a known amount of thiols $(0.4 \mathrm{mg} / \mathrm{ml} \mathrm{GSH}, 5 \mu \mathrm{g} / \mathrm{ml}$ cysteine, $0.2 \mu \mathrm{g} / \mathrm{ml}$ cysteinylglycine, $5 \mu \mathrm{g} / \mathrm{ml} \quad \gamma$-glutamylcysteine and $0.2 \mu \mathrm{g} / \mathrm{ml}$ homocysteine in a solution of $5 \mathrm{mM}$ EDTA) and disulphides $(0.4 \mathrm{mg} / \mathrm{ml}$ GSSG, $1 \mu \mathrm{g} / \mathrm{ml}$ cystine, $0.1 \mu \mathrm{g} / \mathrm{ml}$ cysteinylglycine (oxidised 
form) and $0.1 \mu \mathrm{g}$ homocysteine in a solution of $5 \mathrm{mM}$ EDTA) was analysed.

\section{Statistical analysis}

Statistical analyses from three replicate experiments for treatment comparisons (40-50 eggs per treatment per experiment) were carried out by analysis of variance (ANOVA) and Fisher's protected least significant difference test using the STATVIEW program (Abacus Concepts, Berkeley, CA). Data in the text were expressed as mean \pm SEM. A probability of $p<0.05$ was considered statistically significant.

\section{Results}

When 27 DSH eggs were examined under a phase-contrast microscope after fixation, dehydration and staining, a decondensed sperm nucleus was observed in all eggs. When the tail of penetrated spermatozoa was observed in zona-free DSH eggs, only $12.1 \% \pm 6.9 \%$ of the tail was observed inside of the plasma membrane and $59.8 \% \pm 8.8 \%$ was not yet bound to the membrane $(n=18)$.

Intracellular contents of reduced (GSH) and oxidised glutathione (CSSG) examined by HPLC are shown in Fig. 1. The content of GSH was lower $(P<$ $0.01)$ in $\mathrm{PN}$ eggs $(5.93 \pm 0.10 \mathrm{pmol} / \mathrm{egg})$ when compared with unpenetrated oocytes $(8.50 \pm 0.29$ pmol/egg) and DSH eggs (7.72 $\pm 0.56 \mathrm{pmol} / \mathrm{egg})$. GSSG content was much lower $(p<0.01)$ than that of GSH but did not differ $(p=0.31)$ among experimental groups $(152.6 \pm 74.1 \mathrm{nmol} / \mathrm{egg}$ in unpenetrated eggs, $146.0 \pm 50.0 \mathrm{nmol} / \mathrm{egg}$ in DSH eggs and $39.7 \pm 17.3$ $\mathrm{nmol} / \mathrm{egg}$ in PN eggs). The GSSG/GSH ratio did not

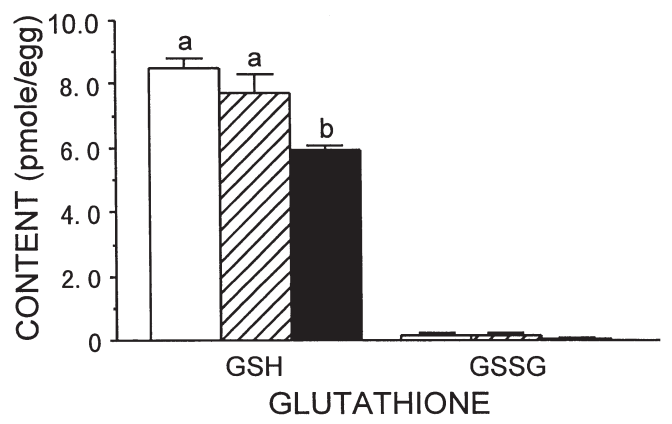

Figure 1 Changes in intracellular content of reduced glutathione (GSH) and glutathione disulphide (GSSG) among unpenetrated oocytes (open columns), penetrated eggs with a decondensed sperm head (DSH eggs) (hatched columns), and zygotes at the pronuclear stage (PN eggs) (black columns). Data from three replicate experiments (40-50 eggs per treatment per experiment) are expressed as mean \pm SEM. Bars with different letters differ $(p<0.05)$. differ among treatment groups $(p=0.40 ; 1.77 \% \pm 0.83 \%$ in unpenetrated eggs, $1.99 \% \pm 0.78 \%$ in DSH eggs and $0.68 \% \pm 0.30 \%$ in PN eggs).

As shown in Fig. 2, there was no difference in the content of reduced cysteinylglycine among experimental groups $(p=0.24)$, but the content of oxidised cysteinylglycine was higher $(p<0.025)$ in PN eggs (40.5 $\pm 11.5 \mathrm{nmol} / \mathrm{egg})$ than unpenetrated oocytes $(6.9 \pm$ $1.5 \mathrm{nmol} / \mathrm{egg})$ and DSH eggs $(10.1 \pm 2.1 \mathrm{nmol} / \mathrm{egg})$. Low contents of cystine were detected in all groups without a significant difference $(p=0.15 ; 34.4 \pm 10.2$ $\mathrm{nmol} /$ egg in unpenetrated eggs, $97.9 \pm 12.6 \mathrm{nmol} / \mathrm{egg}$ in DSH eggs, and $121.2 \pm 44.8 \mathrm{nmol} / \mathrm{egg}$ in PN eggs), although cysteine was not detected in any treatment groups (Fig. 3). Neither reduced nor oxidised forms of $\gamma$-glutamylcysteine were detected in any type of eggs (data not shown).

\section{Discussion}

The tripeptide, glutathione, has many physiological roles such as a storage and transport forms of cysteine, and cellular protection from toxic oxidative stress (Kosower, 1978; Chance et al., 1979; Meister \& Anderson, 1983; Akerboom \& Sies, 1990). Inhibition of glutathione synthesis during oocyte maturation in vitro did not affect the progress of meiosis but delayed the appearance of sperm decondensation in hamsters and pigs (Perreault et al., 1988; Yoshida, 1993). It is believed that the oxidation of reduced glutathione (GSH) is needed to disrupt disulphide bonds of the nuclear protamine of penetrated spermatozoa (Perreault, 1990; Yanagimachi, 1994). In the present study using HPLC with fluorescence detection, the level of GSH in rat eggs was much higher than that of GSSH or other

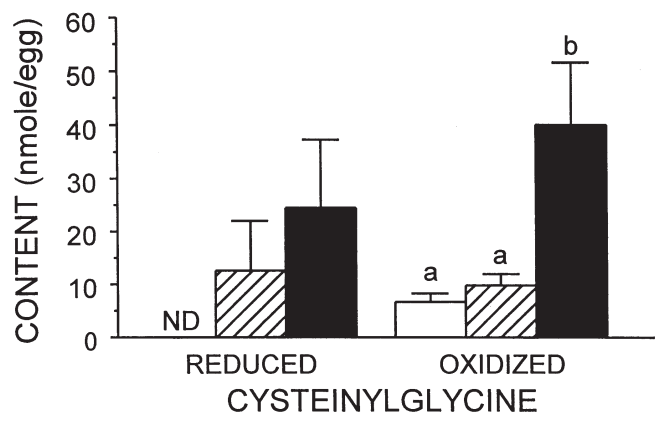

Figure 2 Changes in intracellular content of reduced and oxidised forms of cysteinylglycine among unpenetrated oocytes (open columns), penetrated eggs with a decondensed sperm head (DSH eggs) (hatched columns) and zygotes at the pronuclear stage (PN eggs) (black columns). Data from three replicate experiments (40-50 eggs per treatment per experiment) are expressed as mean \pm SEM. Bars with different letters differ $(p<0.05)$. ND, not detected. 


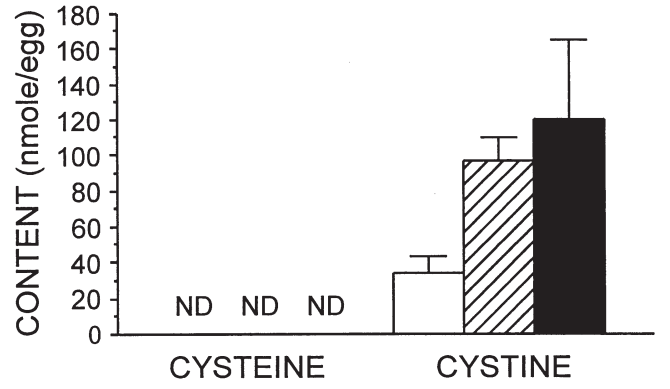

Figure 3 Changes in intracellular content of cysteine and cystine among unpenetrated oocytes (open columns), penetrated eggs with a decondensed sperm head (DSH eggs) (hatched columns) and zygotes at the pronuclear stage (PN eggs) (black columns). Data from three replicate experiments (40-50 eggs per treatment per experiment) are expressed as mean \pm SEM. Bars with different letters differ $(p<0.05)$. ND, not detected.

thiols and a constant GSH/GSSG ratio was maintained during sperm penetration and pronuclear formation. The content of glutathione disulphide is kept at a low level in mouse embryos (Gardiner \& Reed, 1994) and in any cellular system examined to date, because the action of glutathione reductase reduces the oxidised glutathione (Perreault, 1990). The activity of glutathione reductase appears to be maintained at a very high level in rat eggs, resulting in a constant GSH/GSSG ratio during sperm penetration and pronuclear formation even if GSH is oxidised to disrupt disulphide bonds of sperm protamine.

In hamster and pig oocytes, intracellular levels of glutathione containing GSH and GSSG increase during maturation following germinal vesicle breakdown and are lower in fertilised eggs at the pronuclear stage when compared with mature oocytes (Perreault et al., 1984; Yoshida et al., 1993; Funahashi et al., 1995). In porcine eggs, total glutathione (GSH and GSSG) content decreased following sperm penetration and prior to sperm decondensation (Funahashi et al., 1995). In the present study, however, we demonstrated that glutathione content decreased in rat oocytes between sperm decondensation and pronuclear formation. Furthermore, the content of oxidised cysteinylglycine in rat eggs increased at the same time as the glutathione content decreased, although reduced form was not significantly different among treatment groups. Since GGT catalyses the resolution of glutathione to cysteinylglycine in the $\gamma$-glutamyl cycle (Meister et al., 1976; Tate \& Meister, 1981), the activity of $\gamma$-glutatmyl transpeptidase appears to change in oocytes between sperm decondensation and pronuclear formation. There are few studies investigating why oocyte glutathione is decreased during fertilisation. Recently, Funahashi et al. (1996) demonstrated that $\gamma$-glutamyl transpeptidase (GGT) was detected at the surface of the acrosome, connecting piece and midpiece regions of boar spermatozoa. Microinjection of GGT into porcine oocytes matured in vitro induced decreases in both the oocyte glutathione content and the incidence of male pronuclear formation (Funahashi et al., 1996). Since the plasma membrane of the acrosome region is lost during the acrosome reaction, it is quite likely that GGT in the midpiece region is introduced into oocytes at sperm penetration, although, in general, the degradation of GSH occurs extracellularly. In the present study only a small part of the sperm tail was observed inside of the zona-free DSH eggs, whereas almost all the sperm tail had been incorporated in the PN eggs. The decrease in gluthatione levels at a different phase of fertilisation in pig and rat oocytes may be due to a difference in the timing and degree to which the sperm tail is incorporated into the cytoplasm of oocytes.

Cysteinylglycine is degraded into cysteine and glycine by the activity of dipeptidases. In the present study we detected relatively low levels of cysteinylglycine and cystine. Those contents were much lower than the decrease in glutathione content. These data suggest that the activity of dipeptidases is relatively high in rat eggs during fertilisation. It is likely that both cystine and glycine produced from cysteinylglycine by the activity of dipeptidases may be used for protein synthesis in zygotes. Sperm penetration is known to induce changes in the protein profile (Sun et al., 1992). Furthermore, in the present study we could not detect cysteine and $\gamma$-glutamylcysteine although cystine was detected in equal amounts among treatment groups. Glutathione is synthesised by the consecutive action of $\gamma$-glutamylcysteine synthase and GSH synthetase. $\gamma$ Glutamylcysteine synthase, which catalyses the synthesis of $\gamma$-glutamylcysteine from cysteine and glutamic acid, is feedback-inhibited by GSH and appears to be the rate-limiting step in GSH synthesis (Meister, 1995). Glutathione synthesis may not be active in mature rat oocytes and penetrated eggs. GSH content of mouse eggs continues to decrease during fertilisation and early embryonic development (Gardiner \& Reed, 1994).

In conclusion, HPLC with fluorescence detection demonstrates that glutathione content decreases in rat eggs between sperm decondensation and pronuclear formation. At the same time, the content of oxidised cysteinylglycine in rat eggs increased, suggesting that the activity of $\gamma$-glutamyl transpeptidase changes in eggs between sperm decondensation and pronuclear formation.

\section{Acknowledgement}

We would like to thank Dr Billy N. Day and Ms Betty Nichols, University of Missouri-Columbia, for their 
reading and critical advice. This work was supported by a Grant-in-Aid for Scientific Research C (no. 09660303) to H.F. from the Ministry of Education, Science, Sports and Culture of Japan.

\section{References}

Akerboom, T. \& Sies, H. 91990). Glutathione transport and its significance in oxidative stress. In Glutathione: Metabolism and Physiological Functions, ed. J. Vina, pp. 45-55. Boca Raton, FL: CRC Press.

Chance, B., Sies, H. \& Boveris, A. (1979). Hydroperoxide metabolism in mammalian organs. Physiol. Rev. 59, 527-605.

Funahashi, H., Stumpf, T.T., Cantley, T.C., Kin, N.H. \& Day, B.N. (1995). Pronuclear formation and intracellular glutathione content of in vitro-matured porcine oocytes following in vitro fertilization and/or electrical activation. Zygote 3, 273-81.

Funahashi, H., Macathy, Z., Prather, R.S. \& Day, B.N. (1996). $\gamma$-Glutamyl transpeptidase of spermatozoa may decrease oocyte glutathione content at fertilization in pigs. Mol. Reprod. Dev. 45, 485-90.

Gardiner, C.S. \& Reed, D.J. (1994). Status of glutathione during oxidant-induced oxidative stress in the preimplantation mouse embryo. Biol. Reprod. 51, 1307-14.

Kosower, N.S. (1978). The glutathione status of cells. Int. Rev. Cytol. 54, 106-60.

Meister, A. (1995). Glutathione metabolism. In Biothiols, part A, Monothiols and Dithiols, Protein Thiols, and Thiyl Radicals, ed. L. Packer, pp. 3-7. San Diego: Academic Press.

Meister, A. \& Anderson, M.E. (1983). Glutathione. Annu. Rev. Biochem. 52, 11-60.

Meister, A., Tate, S.S. \& Ross, L.L. (1976). Membrane-bound $\gamma$-glutamyl transpeptidase. In The Enzymes of Biological Membranes, ed. A. Martinosi, vol. 3, pp. 315-47. New York: Plenum Press.

Perreault, S.D. (1990). Regulation of sperm nuclear reactiva- tion during fertilization. In Fertilization in Mammals, ed. B.D. Bavister, J. Cumins \& E.R.S. Roldan, pp. 285-96. Norwell, MA: Serono Symposia USA.

Perreault, S.D., Wolff, R.A. \& Zirkin, B.R. (1984). The role of disulfide bond reduction during mammalian sperm nuclear decondensation in vivo. Dev. Biol. 101, 160-7.

Perreault, S.D., Barbee, R.R. \& Slott, V.L. (1988). Importance of glutathione in the acquisition and maintenance of sperm nuclear decondensing activity in maturing hamster oocytes. Dev. Biol. 125, 181-6.

Sun, F.Z., Hoyland, J., Huang, X., Mason, W. \& Moor, R.M. (1992). A comparison of intercellular changes in porcine eggs after fertilization and electroactivation. Development 115, 947-56.

Tate, S.S. \& Meister, A. (1981). $\gamma$-Glutamyl transpeptidase: catalytic, structural and functional aspects. Mol. Cell Biochem. 39, 357-68.

Toyo'oka, T., Uchiyama, S. \& Saito, Y. (1988). Simultaneous determination of thiols and disulfides by high-performance liquid chromatography with fluorescence detection. Anal. Chim. Acta 205, 29-41.

Yanagimachi, R. (1994). Mammalian fertilization. In The Physiology of Reproduction, ed. E. Knobil, J.D. Neill, G.S. Greenwald, C.L. Markert \& D.W. Pfaff, pp. 189-317. New York: Raven Press.

Yoshida, M. (1993). Role of glutathione in the maturation and fertilization of pig oocytes in vitro. Mol. Reprod. Dev. 35, 76-81.

Yoshida, M., Ishigaki, K., Nagai, T., Chikyu, M. \& Pursel, V.G. (1993). Glutathione concentration during maturation and after fertilization in pig oocytes: relevance to the ability of oocytes to form male pronucleus. Biol. Reprod. 49, 89-94.

Zirkin, B.R., Perreault, S.D. \& Naish, S.J. (1989). Formation and function of the male pronucleus during mammalian fertilization. In The Molecular Biology of Fertilization, ed. H. Schatten \& G. Schatten, pp. 91-144. San Diego: Academic Press. 